\title{
In Situ Measurement and Control of the Fermi Level in Colloidal Nanocrystal Thin Films during Their Fabrication
}

\section{Journal Article}

Author(s):

Volk, Sebastian (1); Yazdani, Nuri (1); Yarema, Olesya (1); Yarema, Maksym (1); Bozyigit, Deniz; Wood, Vanessa (1)

Publication date:

2018-12-20

Permanent link:

https://doi.org/10.3929/ethz-b-000313366

Rights / license:

In Copyright - Non-Commercial Use Permitted

Originally published in:

The Journal of Physical Chemistry Letters 9(24), https://doi.org/10.1021/acs.jpclett.8b03283 


\section{In-situ Measurement and Control of the Fermi Level}

\section{in Colloidal Nanocrystal Thin Films during their}

\section{Fabrication}

Sebastian Volk, Nuri Yazdani, Olesya Yarema, Maksym Yarema, Deniz Bozyigit, Vanessa Wood*

*Department of Information Technology and Electrical Engineering, ETH Zurich, Gloriastrasse 35, 8092 Zurich, Switzerland

KEYWORDS: In-situ measurement, electronic structure, Fermi level, nanocrystals, quantum dots

ABSTRACT: In engineering a high-performance semiconductor device, understanding of the Fermi level position is critical. Here, we demonstrate that open-circuit potential (OCP) measurements can be used to quantify the Fermi level in nanocrystal thin films in-situ during their solution-based fabrication. We use this method to study the influence of (1) a metal contact and (2) nanocrystal surface termination on the Fermi level of the nanocrystal film, and find that oxidization or reduction of the nanocrystals as well as surface terminations with dipoles can be used to tune the Fermi level over large energy ranges. Finally, to emphasize the compatibility of the technique with device fabrication, we show that we can use blends of ligands to design the Fermi level landscape in a nanocrystal film. Our work highlights that OCP measurements can be 
used to gain insights into existing device operation and direct further optimization of optoelectronic devices.

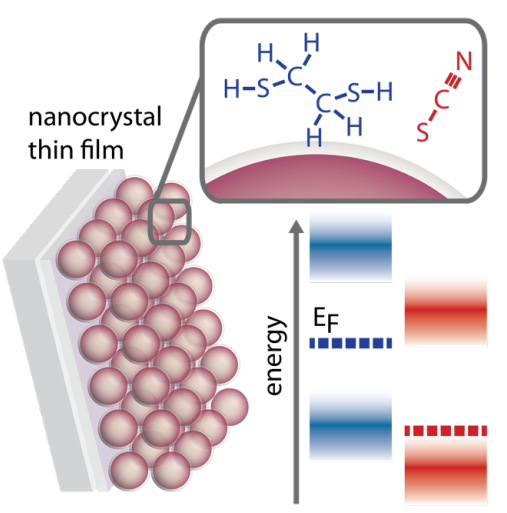


Colloidally-synthesized nanocrystals (NCs) exhibit size-dependent electronic structures, making them attractive for optoelectronic devices. ${ }^{1,2}$ In addition to size control, surface functionalization via ligand exchange provides a means to tune the electronic properties of NC-based thin films. The surface groups determine the electronic coupling between NCs and can strongly influence the electronic structure within thin films, with shifts in the band edges and Fermi level on the order of 1-2 eV. ${ }^{3,4}$ Here, we demonstrate how open-circuit potential (OCP) measurements can be used to quantify the Fermi level in a device during NC thin film fabrication ${ }^{4}$ to facilitate the development of record performing devices. ${ }^{5,6}$

We begin by introducing the concept of surface dipole formation across a metal-semiconductor interface and how a measured voltage difference can be linked to the Fermi energy. Figure 1a illustrates how the electronic structure aligns across a metal-semiconductor interface. Separated by a finite distance, a metal and semiconductor (e.g., here a NC thin film) share the same vacuum level. ${ }^{7-9}$ After contact formation, the electrochemical potential equilibrates through electron transfer from the low work function material to high work function material. ${ }^{10}$ In equilibrium, the redistribution of charge causes a contact potential difference, offsetting the work function difference and shifting the vacuum level. This is often visualized as formation of an interface dipole. If the metal has a larger work function than the semiconductor, the induced surface dipole points away from the metal surface, lowering the vacuum level (Figure 1a, right). Thus, a metal substrate's work function is modified from $\Phi_{1}$ to $\widetilde{\Phi}_{1}$ with respect to the shifted vacuum level after deposition of a semiconductor thin film.

In the case of organic semiconductors deposited on metallic substrates, the shift in work function depends on both charge separation due to charge-transfer across the metal-organic interface as explained above and on the intrinsic molecular dipole of the molecular species itself. ${ }^{11}$ For 
example, self-assembled monolayers (SAMs) of alkanethiols and perfluorinated alkanethiols with opposite intrinsic molecular dipoles have been employed to increase ${ }^{12,13}$ and decrease $\mathrm{e}^{12,14-16}$ the work function of the underlying transition metal substrates. Such shifts can be on the order of $1 \mathrm{eV}$ and beyond as in the case of $\mathrm{Ag}$ (with a work function $\phi_{A g}=4.4 \mathrm{eV}$ ), where surface modified samples are observed to have work functions in a range $\tilde{\phi}_{A g}=3.8 \mathrm{eV}-5.5 \mathrm{eV} .{ }^{17} \mathrm{NC}$ based thin films, like organics, can exhibit both charge-transfer as well as dipoles, due to asymmetry of the NC or its surface functionalization.

Fermi levels in semiconductor thin films and induced work function shifts after their deposition on metals are commonly probed using x-ray and ultraviolet photoelectron spectroscopy (XPS and UPS), ${ }^{4,6,18}$ scanning tunneling spectroscopy (STS), ${ }^{19,20}$ and Kelvin Probe microscopy ${ }^{6}$. As these techniques are best performed in ultra-high vacuum conditions, they are often applied insitu to study thin films deposited in adjacent, connected system and are generally less compatible with solution-fabricated thin films. On the other hand, electrochemical (EC) techniques, which are themselves performed in solution, are an intuitive choice for measuring the electronic structure of $\mathrm{NC}$ thin films. Cyclic voltammetry, ${ }^{21-25}$ (energy-resolved) electrochemical impedance spectroscopy (ER-EIS, EIS), ${ }^{25-27}$ and differential pulse voltammetry ${ }^{28,29}$ have been employed to probe band onsets and quantify the density of states in NC thin films. ${ }^{25}$ While these techniques determine the band onsets, they do not probe the Fermi level, which is important in understanding the electronic state occupation and junctions formation.

Here, we demonstrate that OCP measurements can be used to quantify the Fermi level position in NC thin films during fabrication. To validate the technique, we use the model system of lead sulfide $(\mathrm{PbS}) \mathrm{NC}$ thin films since their band structure has been extensively investigated and engineered to improve device performance. ${ }^{1,2,30}$ We show that both charge transfer to and from 
the NC film (i.e., oxidation and reduction of the NCs) as well as the dielectric nature of the NC film play important roles in determining the Fermi level in a NC film. We then highlight that the OCP measurements can be performed in-situ by integrating it into a dip-coating assembly and using mixtures of surface ligands to engineer different Fermi levels throughout NC films.

In an OCP measurement, differences in electrochemical potential (i.e. differences in the work required to bring a unit charge from vacuum into the system) are measured with a voltmeter between the substrate/NC/electrolyte system (i.e., the working electrode (WE)) and the reference electrode (RE) (Figure 1b). The required amount of work changes with vacuum level shifts at the WE surface. These vacuum level shifts are equivalent to the Fermi level offset of the substrate and $\mathrm{NC}$ thin film before contact formation. Thus, we can quantify the Fermi level in the $\mathrm{NC}$ thin film by tracking the electrochemical potential difference of a modified WE with respect to a RE.

The electrolyte consists of a salt (e.g. $100 \mathrm{mM}$ Tetrabutylammonium perchlorate (TBAP)) dissolved in anhydrous acetonitrile (anh $\mathrm{ACN}$ ). $300 \mu \mathrm{M}$ ferrocene $(\mathrm{Fc})$ is used to set the electrochemical potential of the supporting electrolyte. Indium tin oxide (ITO) in this Fccontaining electrolyte is used as a pseudo-reference electrode with an absolute electrochemical potential $E_{a b s}^{I T O}=4.9 \mathrm{~V}$ versus vacuum (Experimental Section), as determined through calibration against an $\mathrm{Ag} / \mathrm{Ag}^{+}$reference electrode. TBAP and $\mathrm{Fc}$ concentrations are kept to a minimum to limit contamination of the NC thin film, but are large enough that ITO vs ITO OCP measurements show quickly vanishing (within seconds) measured voltage differences $(<10 \mathrm{mV})$ (Figure 1c left, Supporting Information SI 1). Figure 1c (right) shows the effect of a single monolayer of $1.3 \mathrm{eV}$ band gap (as determined by the $1^{\text {st }}$ exciton absorption feature in hexane) $\mathrm{PbS}$ NCs terminated with 1,2-Ethanedithiol (EDT) on ITO, deposited at ambient conditions. 
Equilibration processes are visible within the first $\sim 5 \mathrm{~s}$. After this time, a stable voltage difference of about $-285 \mathrm{mV}$ with respect to ITO is observed.

Using the voltage difference measured with OCP, we can understand the energetic structure at an ITO/PbS:EDT interface (Figure 1d). Because ITO has a work function larger than that of the $\mathrm{PbS} \mathrm{NCs}$, the NCs oxidize, leading to charge separation and a surface dipole pointing away from the ITO surface. This results in a Fermi level of $E_{F}^{\mathrm{ITO} / \mathrm{PbS}: \mathrm{EDT}}=-(4.9 \mathrm{eV}-0.285 \mathrm{eV})=$ $-4.615 \mathrm{eV}$ in the $\sim 5 \mathrm{~nm}$ thick film of PbS:EDT on ITO.

To confirm that oxidation or reduction of NCs and the resulting surface dipole at the metal-NC interface plays a key role in determining the Fermi level in a NC thin film, we investigate the same NC thin film on a series of substrates with different work functions. We select commonly used contacts in $\mathrm{NC}$ devices as substrates: ITO, ITO/Molybdenum Oxide $\left(\mathrm{MoO}_{3}\right), \mathrm{Al}$, and $\mathrm{Al} / \mathrm{LiF}$. On each of these substrates, we deposit a $20 \mathrm{~nm}$ PbS:EDT film, performing OCP measurements after every dip coating cycle (approximately every $4-5 \mathrm{~nm}$, based upon atomic force microscopy (AFM)). The values (and error bars) shown in Figure 2a represent the mean and standard deviations of 20 seconds-long OCP time traces, recorded after $20 \mathrm{~s}$ to allow the system to first reach equilibrium. We measure substrate-dependent absolute potentials of $E_{a b s}^{I T O}=$ $4.9 \mathrm{~V}, E_{a b s}^{I T O / \mathrm{MoO}_{3}}=4.97 \mathrm{~V}, E_{a b s}^{A l / L i F}=3.54 \mathrm{~V}$, and $E_{a b s}^{A l}=3.97 \mathrm{~V}$, following the trend of literature values of their respective work functions (note, that we rule out the influence of standard potentials in the sense of mixed potential theory, Supporting Information SI 2). ${ }^{31-38}$. In contrast to ITO and $\mathrm{ITO} / \mathrm{MoO}_{3}$ giving rise to $\mathrm{NC}$ oxidation, $\mathrm{Al}$ or $\mathrm{Al} / \mathrm{LiF}$ have work functions lower than that of the NCs such that the NCs are reduced and the surface dipoles point toward the metal substrate. The case of PbS:EDT on $\mathrm{Al} / \mathrm{LiF}$ is shown in Figure 2b. First, in line with 
previous reports, ${ }^{37,38}$ a $3 \mathrm{~nm}$ thin $\mathrm{LiF}$ layer deposited on $\mathrm{Al}$ lowers the vacuum level by $430 \mathrm{meV}$, creating a dipole at the $\mathrm{Al} / \mathrm{LiF}$ interface. ${ }^{35}$ Upon PbS:EDT deposition, the NCs are reduced and the vacuum level increases by $250 \mathrm{meV}$, corresponding to a Fermi level of $-3.75 \mathrm{eV}$ in the $\mathrm{NC}$ thin film.

Figure 2a shows that a single layer of NCs is sufficient to support a dipole of several hundred meV. About $80 \%$ of the overall measured voltage drop occurs within the first monolayer (measured voltage after first dipcoating cycling is $-285 \mathrm{mV}$ and after 5 cycles it is $-360 \mathrm{mV}$ ). Transfer of a single electron per NC between the substrate and first NC monolayer layer leads to an estimated vacuum level shift of $325 \mathrm{meV}$ (Supporting Information SI 3) consistent with the potential differences observed in Figure 2a. Density functional theory (DFT) calculations show that a positively or negatively charged $\mathrm{PbS} \mathrm{NC}$ with thiol termination in vacuum exhibits a shift in energy levels on the order of $950 \mathrm{meV}$ relative to a neutral NC (Figure 2c), and, due to charge screening effects, we expect the magnitude of the shift in energy levels to be smaller in a NC solid. Our measurements and calculations highlight that, when charge is localized on individual $\mathrm{NCs}$, it is feasible to have dipole-induced shifts of several $100 \mathrm{meV}$. This may account for the contact metallurgy effects observed in transistors and diodes (Supporting Information SI 4).

The Fermi level of a NC thin film is also dependent on the surface-termination of the NCs. Surface-termination induced shifts of the electronic structure of capped NCs have been attributed either to the intrinsic dipole moment of the linker species itself ${ }^{39}$ or to the NC-ligand interface dipole,${ }^{19}$ and DFT calculations predict a combination of both. ${ }^{4}$ We perform OCP measurements on $20 \mathrm{~nm}$-thick PbS NC thin films on ITO with 1,2-EDT, 3-Mercaptopropionic acid (MPA), 1,4Butanedithiol (BDT), and Ammonium thiocyanate $\left(\mathrm{NH}_{4} \mathrm{SCN}\right)$ ligands, and measure Fermi levels of $\quad E_{F}^{I T O / P b S: E D T}=-4.56 \mathrm{eV}, \quad E_{F}^{I T O / P b S: M P A}=-4.59 \mathrm{eV}, \quad E_{F}^{I T O / P b S: B D T}=-4.64 \mathrm{eV}, \quad$ and 
$E_{F}^{I T O / P b S: \mathrm{NH}_{4} S C N}=-4.89 \mathrm{eV}$ (Figure 3a). These findings are consistent with previous studies (Supplementary Information SI 5), highlighting that a solution-based electrochemical methodology can provide comparable information to that obtained by high-vacuum analytical techniques.

All the ligands studied attach via sulfur atom bonding to a $\mathrm{Pb}$ surface atom with a reported ${ }^{4}$ interface dipole between the $\mathrm{NC}$ and the $\mathrm{S}$ atom of $1.5-1.9 \mathrm{D}$; however, they differ in their effective molecular dipole moment. Assuming a primarily monodendate attachment, EDT and BDT exhibit a zero intrinsic dipole moment vertical to the $\mathrm{NC}$ surface due to molecular symmetry. Even though MPA as molecular monomer has a reported dipole moment of $2.25 \mathrm{D},{ }^{40}$ organic carboxylic acids are known to favor dimerization via hydrogen-bonding, ${ }^{41,42}$ which would result in a zero net dipole moment. The zero net dipole moment explains the similarity in the Fermi levels $\left(E_{F}^{I T O / P b S: E D T}=-4.56 \mathrm{eV}, \quad E_{F}^{I T O / P b S: M P A}=-4.59 \mathrm{eV}, \quad E_{F}^{I T O / P b S: B D T}=\right.$ $-4.64 \mathrm{eV}$ ). In contrast, $\mathrm{SCN}^{-}$surface termination (via $\mathrm{NH}_{4} \mathrm{SCN}$ treatment, see Experimental Section) results in a much lower Fermi level $\left(E_{F}^{I T O / P b S: N H_{4} S C N}=-4.89 \mathrm{eV}\right)$. SCN, ${ }^{-}$with a reported dipole of $1.78 \mathrm{D}^{43}$ (pointing towards the $\mathrm{PbS}$ surface due to the strong electronegativity of $\mathrm{N}$ ) lowers the electronic structure. Indeed, we confirm this by measuring the $\mathrm{CB}$ and VB onsets of $\mathrm{SCN}^{-}$-capped $\mathrm{PbS} \mathrm{NCs}$ through ER-EIS ${ }^{25}$ under nitrogen conditions and quantify a mean CB and VB shift of $202 \mathrm{meV}$ compared to EDT (Figure 3b). The band onsets agree with XPS and UPS measurements, ${ }^{4}$ and the position of the Fermi level determined here with OCP is consistent with STS studies. ${ }^{44}$ The lowering of the energy levels translates into a surface-dipole induced shift of $0.11 \mathrm{eV} / \mathrm{D}$, equivalent to XPS studies by Kroupa et al. reporting $0.13 \mathrm{eV} / \mathrm{D} .{ }^{3} \mathrm{We}$ further observe that the Fermi level lies in close vicinity of the VB, in contrast to the Fermi level in PbS:EDT NC film, which is centered within the bandgap. We attribute this to the fact that 
thiocyanates are capable of decomposing at surfaces with strong affinity to $\mathrm{S}$ atoms, via cleavage of the S-C bond and dissociation to sulfide and cyanide monolayers. ${ }^{45-48}$ In contrast to EDT, where functional thiol groups bind to $\mathrm{Pb}$ surface atoms, the thiocyanate dissociation could partially result in single sulfur atoms being effectively incorporated into the $\mathrm{PbS}$ matrix, lowering the cation to anion ratio of the NC. Such a stoichiometry change could explain the positioning of the Fermi level close to the valence band onset as proposed in transport study measurements ${ }^{49,50}$ and predicted from theory. ${ }^{51}$ The decreasing Fermi level with increasing film thickness (Figure 3a) is consistent with more sulfur incorporation for an increasing number of dipcoating cycles. This highlights that NC is also a means to control the Fermi level position within the NC thin film.

In summary, a number of factors must be considered when designing an interface in a NC device. First, the workfunction difference between the metal and the NC thin film determines the charge state of the NC layer. The dielectric environment of the NCs - determined by the NCs themselves, their packing, and their ligands - determines how this charge is screened. The presence of intrinsic dipoles from the ligands can result in further shifts in the energy level. It can further be expected that a NC-film thickness dependence of the Fermi level may be observed, trending towards the Fermi-level of the NC material/ligand couple in vacuum. This thickness dependence will depend for example on how charge reorganizes over a given thickness (dependent on the electronic coupling between NCs and the energetic disorder of the thin film) as well as chemical changes to the film during deposition (e.g., deposition in air can lead to progressive oxidation).

$\mathrm{NC}$ thin films are typically processed from solution in a layer-by-layer procedure using spin coating, dipcoating, or spray coating. ${ }^{52-54} \mathrm{We}$ integrate OCP measurements seamlessly into the 
dipcoating procedure to monitor the potential landscape during device fabrication (Experimental Section and photograph in Supporting Information SI 6). To assess whether this technique can be used in-situ, we look at the effect of successive OCP measurements on the measured Fermi level values and on the subsequent device performance. First, we determine that after five subsequent measurement repetitions (in the extreme case without any sample rinsing), the OCP deviations are on the order of $30 \mathrm{mV}$ (Supporting Information SI 7). Second, we analyze the impact of repetitive thin film exposure to the measurement environment on the performance of heterojunction SCs and find that the power conversion efficiency varies by less than 10\% (Supporting Information SI 8). We note that selection of an electrolyte that does not interact strongly with the film is important (usage of Tetrabutylammonium hexafluorophosphate instead of Tetrabutylammonium perchlorate reduces the impact on the device performance parameters). Taken together, these results indicate that if a relatively non-interacting electrolyte is selected and if appropriate rinsing protocols during device fabrication are followed, this technique can be used in-situ, parallel to device fabrication.

Having shown that OCP measurements allow probing the electronic structure of NC thin films during fabrication, we demonstrate its potential to track the Fermi level within a several hundred nanometer thick NC film. Here we engineer the Fermi level by using combinations of EDT and $\mathrm{NH}_{4} \mathrm{SCN}$ ligands. First, we investigate how the Fermi level shifts across an abrupt junction between EDT and $\mathrm{NH}_{4} \mathrm{SCN}$ cross-linked PbS films. We deposit a $40 \mathrm{~nm}$ PbS:EDT film on ITO, followed by $60 \mathrm{~nm}$ PbS: $\mathrm{NH}_{4} \mathrm{SCN}$ and measure the Fermi level approximately every $20 \mathrm{~nm}$ (Figure 4a). The Fermi level as a function of position $x$ in the film can be approximated using an exponential power law $E_{F}(x)=c * e^{-\frac{x}{x_{0}}}+E_{0}$ with a characteristic length of $x_{0}=13 \mathrm{~nm}$, meaning that the Fermi level decays (or grows) to $37 \%$ of the final value across $\sim 3-4$ layers of 
NCs.

To control the Fermi level, we mix the two ligand exchange solutions in different ratios R, with $\mathrm{R}=0 \%$ corresponding to a $\mathrm{NH}_{4} \mathrm{SCN}$ ligand exchange solution and $\mathrm{R}=100 \%$ corresponding to an EDT ligand exchange solution. Figure 4b shows a linear-logarithmic dependence of the Fermi level on R. This calibration curve can be used to design a linear $\mathrm{E}_{\mathrm{F}}$ sweep throughout a NC thin film. Based on our finding of a characteristic length of $13 \mathrm{~nm}$, we deposit five $20 \mathrm{~nm}$ layers with mixing ratios $\mathrm{R}$ of $0 \%, 1.7 \%, 8.4 \%, 38.8 \%$ and $100 \%$ (see intersection of equidistantly spaced horizontal gray lines with calibration curve in Figure $\mathbf{4 b}$ ) to increase $\mathrm{E}_{\mathrm{F}}$ from $4.91 \mathrm{eV}$ to $4.65 \mathrm{eV}$ (Figure 4c). Furthermore, it is possible to create a ratchet-style energy level structure. We start with a $20 \mathrm{~nm}$ thick layer of $\mathrm{PbS}: \mathrm{NH}_{4} \mathrm{SCN}$ on ITO and progressively increase the EDT content in three successive $20 \mathrm{~nm}$ thin films (using ligand exchange solutions with mixing ratios $\mathrm{R}=2.6 \%, 27 \%$ to $100 \%$ ). This sweep can be repeated multiple times as shown in Figure $4 \mathbf{d}$. This in-situ character allows for precise tuning of the electronic structure and matching to contact metallurgy (Supporting Information SI 4).

In summary, we demonstrate OCP measurements as a method to quantify the Fermi level position in $\mathrm{NC}$ thin films. Unlike vacuum-based techniques for quantifying electronic structure, this electrochemical technique is well suited for NC-based thin films as it allows for in-situ assessment during the solution-based thin film fabrication. We show that oxidation and reduction of a single NC layer can lead to dipoles, giving rise to vacuum level shifts on the order of several hundreds of meV. Surface functionalization tunes the dielectric environment, and, by mixing ligand species, we can achieve a preselected Fermi level landscape within the NC thin film. This measurement technique and the insights into charge reorganization within $\mathrm{NC}$ thin films can be leveraged to further optimize electronic bandstructures in optoelectronic devices. 


\section{Methods:}

Chemicals: Acetonitrile (anh. ACN, anhydrous, 99.8\%), 1,2-Ethanedithiol (EDT, $\geq 98.0 \%$ ), 1,4Butanedithiol (BDT, $\geq 97.0 \%$ ), 3-Mercaptopropionic acid (MPA, $\geq 99 \%$ ), Ferrocene (Fc, 98\%), Tetrabutylammonium perchlorate (TBAP, $\geq 98.0 \%$ ), Tetrabutylammonium tetrafluoroborate (for electrochemical analysis, $\geq 99.0 \%$ ), Ammonium hexafluorophosphate (99.99\% trace metal basis), Tetraethylammonium hexafluorophosphate (for electrochemical analysis, $\geq 99.0 \%$ ),

Tetrabutylammonium hexafluorophosphate (for electrochemical analysis, $\geq 99.0 \%$ ), Tetrahexylammonium hexafluorophosphate (gravimetric, $\geq 97.0 \%$ ) are purchased from SigmaAldrich. N-Hexane (95+\%, extra pure, Acros Organics) and Ammonium thiocyanate $\left(\mathrm{NH}_{4} \mathrm{SCN}\right.$, $+99 \%$, extra pure, Acros Organics) are purchased from Fisher Scientific.

Synthesis of the NCs: The synthesis of PbS NCs is carried out using the hot injection method according to Hines and Scholes. ${ }^{55}$ To prepare PbS NCs with $1.3 \mathrm{eV}$ band gap, $1.8 \mathrm{~g}$ of $\mathrm{PbO}$ is mixed with $5 \mathrm{ml}$ of oleic acid and $75 \mathrm{ml}$ of 1-octadecene in a three-neck flask. At $140{ }^{\circ} \mathrm{C} 40 \mathrm{ml}$ of $0.1 \mathrm{M}$ hexamethyldisilathiane solution in octadecene is quickly injected to the purified lead oleate. The reaction mixture is cooled down to $90{ }^{\circ} \mathrm{C}$ and kept at this temperature for another 5 min. Obtained PbS NCs are purified three times using a mixture of hexane and ethanol and centrifugation. The cleaned NCs are dispersed in hexane at a concentration of $5 \mathrm{mg} / \mathrm{ml}$.

Substrate preparation: ITO substrates are cleaned through two sonication steps (5 min each) in Mucasol solution (10 vol\%), deionized water and acetone followed by a 5 min immersion in hot isopropanol, followed by oxygen plasma cleaning. For other substrates than ITO, metals are evaporated on top of ITO. Electrical connection legs (Ossila, E241) are clipped onto the substrate for connection to external circuits. A drop of silver paste is applied on the contact area of the 
connection legs/ substrate and backed-out for $30 \mathrm{~min}$ at $100{ }^{\circ} \mathrm{C}$. All metallic parts are isolated with PDMS to only expose an approximately $1 \mathrm{~cm}^{2}$ window to the electrolyte solution. PDMS is cured for $60 \mathrm{~min}$ at $120^{\circ} \mathrm{C}$.

Solution preparation: $300 \mu \mathrm{M}$ Ferrocene and $100 \mathrm{mM}$ Tetrabutylammonium perchlorate or 100 $\mathrm{mM}$ Tetrabutylammonium hexafluorophosphate in anhydrous acetonitrile are prepared in a nitrogen glovebox and exposed to air just before usage.

Thin film fabrication: Thin films are fabricated using a programmable and custom-built, automated dipcoater setup at ambient conditions or in nitrogen glovebox (if OCP measurements are paired with ER-EIS). Films are built up by immersing the substrate into a $5 \mathrm{mg} / \mathrm{ml}$ solution of PbS NCs in Hexane followed by a crosslinking step in a $5 \mathrm{mM}$ EDT (or alternatively $5 \mathrm{mM}$ BDT, $5 \mathrm{mM}$ MPA) solution in anh. ACN, or $30 \mathrm{mM} \mathrm{NH}_{4} \mathrm{SCN}$ in anh. Methanol. A third rinsing step washes-off excess ligands. Typically, layers of $4 \mathrm{~nm}$ thickness are deposited in one cycle. Atomic force microscopy analysis reveals the NC films to be continuous. This is confirmed by the ER-EIS measurement, where the electronic structure of the NC film, not the underlying substrate is observed.

Thin film potential measurement: Potential differences are measured with a Keithley 2602A SMU between reference electrode and the working electrode, formed by NC thin film deposition. In addition to the three-step dip-coating process, we add four more steps (4. additional rinsing, 5. potential measurement, 6. \& 7. rinsing in anh. $\mathrm{ACN}$ ) to automate and migrate the potential measurements into the fabrication line. After immersing the working electrode into the electrolyte, we allow the system $20 \mathrm{~s}$ to reach an equilibrium state and we record time traces over $20 \mathrm{~s}$ to calculate mean values and standard deviations. All measurements are performed under 
ambient conditions, except for the OCP and ER-EIS datasets in Figure 3b due to the necessity of an oxygen and humidity-free measurement environment for impedance measurements under applied potentials.

Energy-resolved electrochemical impedance spectroscopy: Charge-transfer between ITO and a $\mathrm{NC}$ thin film (same sample fabrication as for OCP measurements) is analyzed using impedance measurements with a perturbation amplitude $E_{a}=25 \mathrm{mV}$ in the frequency range from $1 \mathrm{kHz}$ to 3 $\mathrm{Hz}$ at $2 \mathrm{pts} . / \mathrm{dec} a d e$ in logarithmic spacing in the potential range $\mathrm{OCP} \rightarrow-1.7 \mathrm{~V}$ vs $\mathrm{Ag} / \mathrm{Ag}^{+}(+0.5$ $\mathrm{V}$ vs $\mathrm{Ag} / \mathrm{Ag}^{+}$, fresh samples for each measurement) with a typical energy-resolution of $10 \mathrm{mV}$. A standard Randles circuit consisting out of double layer capacitance in parallel to a chargetransfer resistance, both in series to a series resistance, is used to analyze the charge-transfer event. The inverse charge-transfer resistance is approximated with Gaussian distributions (accounting for the energy spread of the first state) and their means taken as band onsets. ${ }^{25}$

Assignment of electrochemical to physical scale: The relationship between absolute $E_{a b s}^{M}$ and the relative single electrode $E_{\text {rel }}^{M}$ potentials can be written as: ${ }^{56}$

$$
E_{a b s}^{M}=E_{r e l}^{M}+\kappa,
$$

where the constant term $\kappa$ depends on the chosen reference system. To match semiconductor energy levels with the electrochemical potential scale, we follow Trasatti ${ }^{57}$ and define an electron in vacuum just outside the electrolyte phase as a reference point matching the origins of the physical and electrochemical scales. Thus, the Fermi energy of the $\mathrm{Ag} / \mathrm{Ag}^{+}$redox-system $E_{F}^{A g / A g+}$ can be expressed as: 


$$
-E_{F}^{A g / A g+}=e E_{a b s}^{A g / A g+}=e\left(E_{S H E}^{A g / A g+}+\mathrm{E}^{0}\left(H^{+} / H_{2}\right)\right)
$$

with the relative potential of the $\mathrm{Ag} / \mathrm{Ag}^{+}$pair $E_{S H E}^{A g / A g+}=0.542 \mathrm{~V}$ versus the standard hydrogen electrode (SHE) ${ }^{58}$ the elementary charge $e$, and the absolute electrode potential of $\operatorname{SHE~E}^{0}\left(\mathrm{H}^{+} /\right.$ $\left.H_{2}\right)$. The reported values for the absolute SHE potential span 4.73 to $4.44 \mathrm{~V}^{56,57,59}$ and $\mathrm{E}^{0}\left(\mathrm{H}^{+} /\right.$ $\left.H_{2}\right)=4.5 \mathrm{~V}$ is chosen in this study. The negative sign in front of $E_{F}^{A g / A g+}$ comes from the fact that the physical scale points into opposite direction as the absolute electrochemical scale. Electrode potentials: We emphasize that we are not measuring the redox potential associated with ion transfer to or from the film of interest (i.e., the working electrode (WE)), which would not necessarily correlate with its work function. Instead, the electrochemical potential of the $\mathrm{Fc} / \mathrm{Fc}^{+}$couple at the electrode surface is measured. Due to the much larger carrier concentration in the ITO (both forming RE and WE), the electrochemical potential of the $\mathrm{Fc} / \mathrm{Fc}^{+}$couple locally equilibrates with the Fermi levels of the metal.

Estimation of surface dipole due to charge transfer event: A PbS:EDT (3.1 nm diameter) inter$\mathrm{NC}$ spacing $\mathrm{r}_{\text {inter }}=1.43 \mathrm{~nm}$ has been reported in a previous study ${ }^{25}$ based upon small-angle X-ray scattering in agreement with literature. ${ }^{60}$ Nanoparticle radius $\mathrm{r}_{\mathrm{NC}}$ is determined via $1^{\text {st }}$ exciton absorption peak in hexane. PbS:EDT NC networks are reported to from BCC superlattice with the [100] facet parallel to the substrate ${ }^{60}$ which simplifies the effective area of a NC to (NC-NC distance $)^{2}$.

DFT calculations: The atomistic model employed for the $\mathrm{r}=\sim 1.2 \mathrm{~nm}$ PbS NCs were identical to those used previously, ${ }^{25,61,62}$ constructed following the atomistic model proposed by Zherebetskyy et al. ${ }^{63}$ In place of the more computationally demanding ethanedithiol ligands, we used methanethiol (mth) ligands, which capture the chemistry of the $\mathrm{Pb}-\mathrm{S}$ bond. The final $\mathrm{NC}$ 
had $N_{P b}=199$ lead atoms, $N_{S}=140$ sulfur atoms, and $N_{L}=118$ ligands. Electronic structure calculations and geometry optimizations are performed within the $\mathrm{CP} 2 \mathrm{~K}$ program suite utilizing the quickstep module. ${ }^{64} \mathrm{~A}$ dual basis of localized Gaussians and plane-waves, ${ }^{65}$ with a $300 \mathrm{Ry}$ plane-wave cutoff, Double-Zeta-Valence-Polarization (DZVP), ${ }^{66}$ Goedecker-Teter-Hutter pseudopotentials $^{67}$ for core electrons, and the Perdew-Burke-Ernzerhof (PBE) exchange correlation functional are used. Convergence to $10^{-8}$ in Self-Consistent Field calculations is always enforced. For both the structure and the potential, non-periodic boundary conditions are employed, which is solved using the Wavelet Poisson solver ${ }^{68,69}$ A cubic unit cell of dimensions $4 \mathrm{~nm} \times 4 \mathrm{~nm} \times 4 \mathrm{~nm}$ was employed. Geometry optimization is performed with the Quickstep module utilizing a Broyden-Fletcher-Goldfarb-Shannon (BFGS) optimizer, with maximum force of 24 $m e V \AA^{-1}$ is used as convergence criteria. The charge state of the NC was achieved by adding/removing an electron from the system. All atoms in all systems are relaxed. 

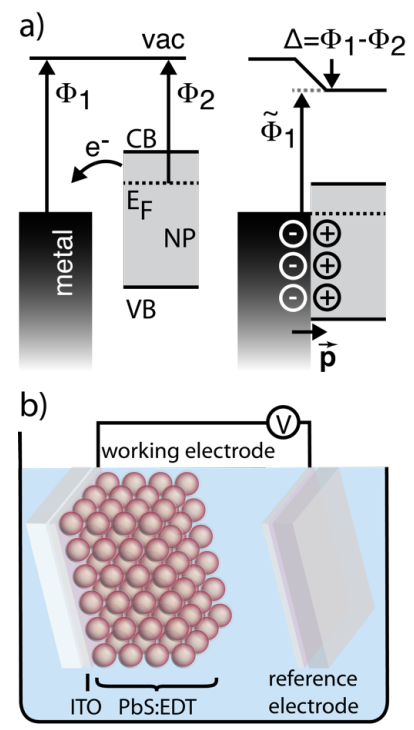

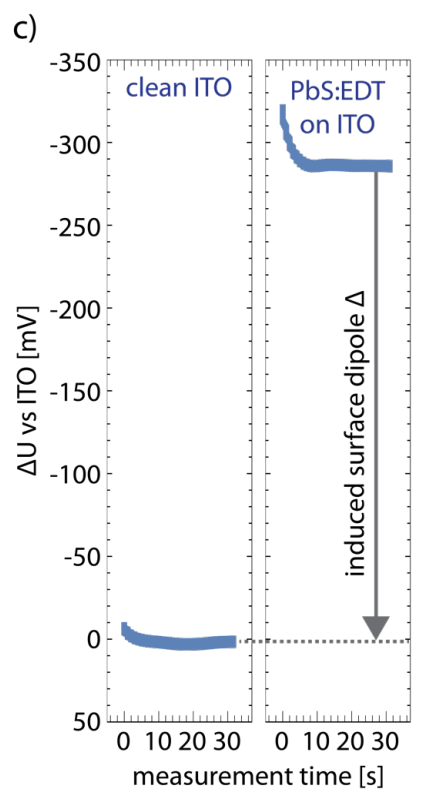

d)
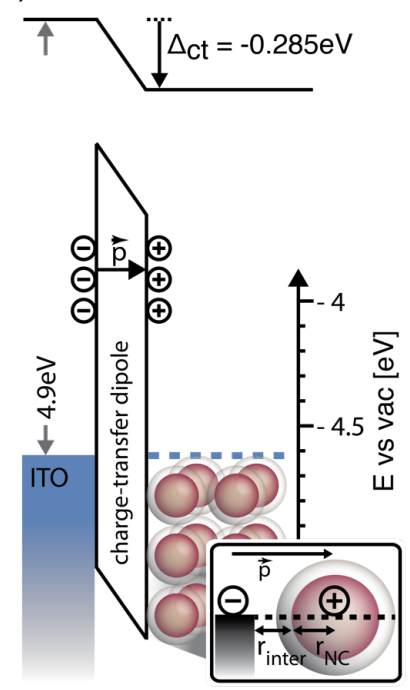

Figure 1. Principle of open-circuit potential (OCP) measurements. a) Schematic of a metalsemiconductor interface. Left: metal and semiconductor a finite distance apart. Here, the metal exhibits a larger work function $\phi_{1}$ with respect to the surface vacuum level than the semiconductor. The semiconductor Fermi level $\left(\mathrm{E}_{\mathrm{F}}\right)$, conduction $(\mathrm{CB})$ and valence band $(\mathrm{VB})$ are indicated. Right: in contact, Fermi level equilibration between the solids takes place through electron charge-transfer from the semiconductor to the metal. As a result, an electric double layer forms, reflected in a surface vacuum level step $\Delta$, equivalent to the difference $\phi_{1}-\phi_{2}$ between the metal and semiconductor work functions before they are brought into contact. The electric double layer can be interpreted as a surface dipole $\mathbf{p}$ pointing away from the metal surface, that lowers its work function to $\widetilde{\phi}_{1}$. This sketch shows the interface and does not include band bending (Supporting Information, SI 2). b) In an OCP measurement, the electrochemical potential differences between a working electrode (the film under measurement such as $\mathrm{PbS}$ nanocrystals (NCs) terminated with ethanedithiol (EDT) ligands on ITO, immersed in electrolyte) and the reference electrode (an ITO film) are measured at open-circuit conditions. c) 
OCP time traces of (left) ITO versus ITO (showing a vanishing potential difference) and (right) NC film on ITO substrates versus ITO showing a $-285 \mathrm{mV}$ potential differences reflective of a surface dipole formation between the PbS and the ITO. d) Bandstructure of a PbS:EDT thin film deposited on ITO. The Fermi level is deduced from the OCP measurement results in panel c). Inset: for quantitative assessment of the magnitude of dipole $\mathrm{p}$ and surface vacuum level shift, we assume charges to be separated by the sum of the interparticle distance $r_{\text {inter }}$ and nanoparticle radius $\mathbf{r}_{\mathrm{NC}}$. The surface dipole can be explained by charge-transfer of 1 electron per NC between substrate and the first NC layer. 


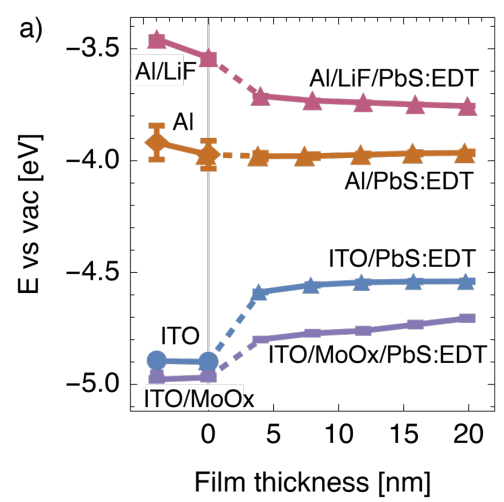

b)
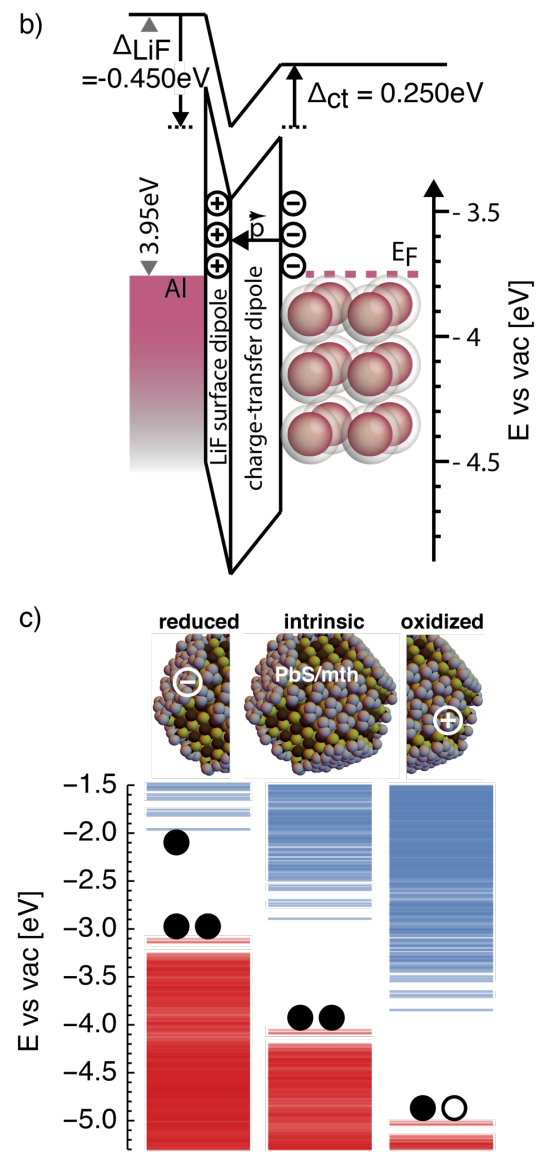

Figure 2. Effect of nanocrystal (NC) oxidation or reduction on Fermi level. a) Fermi levels measured before and during deposition of a PbS:EDT NC thin films on substrates typically used in NC-based optoelectronic devices. b) Schematic illustration of surface dipoles formed after deposition of a $3 \mathrm{~nm}$ thin $\mathrm{LiF}$ layer (which decreases $\mathrm{Al}$ work function by $450 \mathrm{meV}$ ) and deposition of $20 \mathrm{~nm}$-thick $\mathrm{PbS}$ :EDT, where reduction of the $\mathrm{NC}$ thin film gives rise to a surface 
dipole pointing towards the substrate and increases the vacuum level by $250 \mathrm{meV}$. c) Density functional theory on a thiol-terminated $\mathrm{PbS} \mathrm{NC}$ in vacuum shows that an excess negative or positive charge (i.e., reduction or oxidation of the NC) shifts the energy levels by $950 \mathrm{meV}$ compared to that of a neutral NC (center). This shift decreases by a factor of three for simulations performed on NC networks, indicating that oxidation and reduction of a single $\mathrm{NC}$ layer can explain Fermi level shifts as seen in a). 
a)

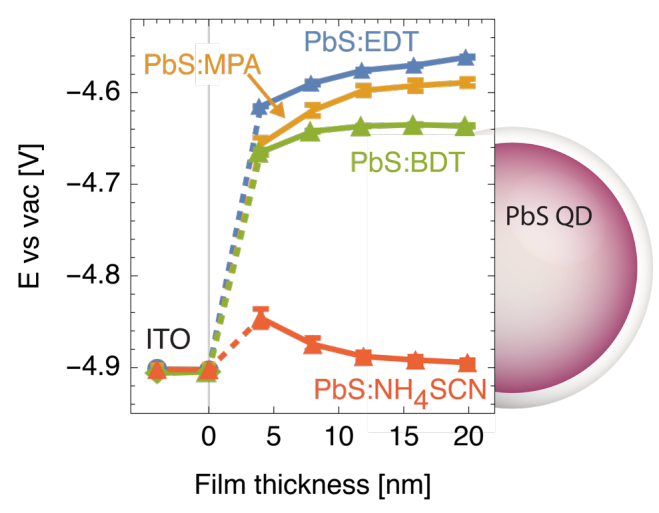

1,2-Ethanedithiol

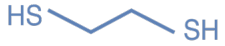

3-Mercaptopropionic

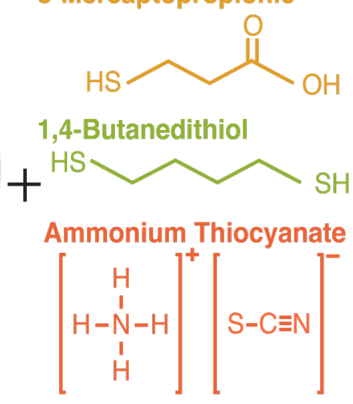

b) -3.8

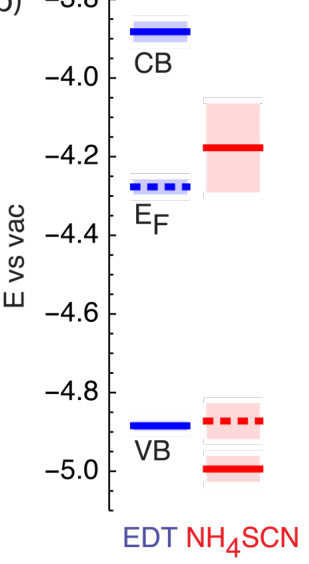

Figure 3. Effect of surface termination. a) Fermi levels determined from open circuit potential (OCP) measurements on ITO and during subsequent deposition of $\mathrm{PbS}$ nanocrystal films using ethanedithiol (EDT), mercaptopropionic acid (MPA), and butanedithiol (BDT), and Ammonium thiocyanate $\left(\mathrm{NH}_{4} \mathrm{SCN}\right)$ as ligands. Schematics of these surface terminations are shown on the right. b) Bandstructure of $\mathrm{PbS}: \mathrm{EDT}$ and $\mathrm{PbS}: \mathrm{NH}_{4} \mathrm{SCN}$ thin films fabricated and measured under nitrogen conditions. Fermi levels are obtained from OCP measurements and conduction band (CB) and valence band (VB) onsets are determined from energy-resolved electrochemical impedance spectroscopy measurements. 

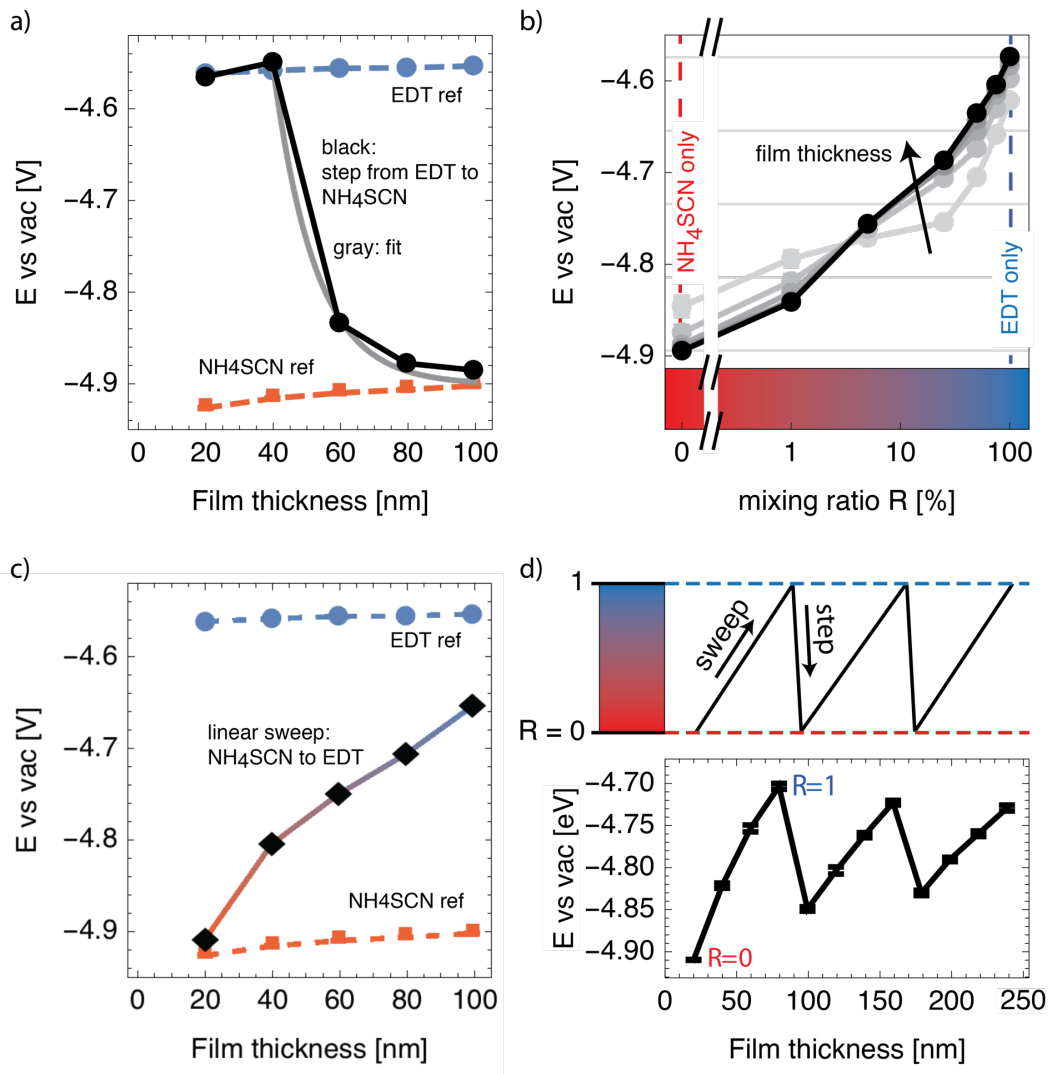

Figure 4. Controlling the Fermi level through ligand mixtures. a) Fermi level measured every $20 \mathrm{~nm}$ for a $40 \mathrm{~nm}$ thin film with an EDT crosslinked solution followed by a $60 \mathrm{~nm}$ film with $\mathrm{NH}_{4} \mathrm{SCN}$ cross linker solution (black). The data points are fit with an exponential curve (gray) indicating a characteristic length scale of $\mathrm{x}_{0}=13 \mathrm{~nm}$. The Fermi level for a $100 \mathrm{~nm}$ think $\mathrm{PbS}$ crossed linked with EDT (blue dashed) or $\mathrm{NH}_{4} \mathrm{SCN}$ (red dashed) are also provided. b) Using different mixtures of the two stock ligand exchange solutions (5.5 mM EDT in anh. ACN or 30 $\mathrm{mM} \mathrm{NH}_{4} \mathrm{SCN}$ in anh. $\mathrm{MeOH}$, respectively) different nanocrystal (NC) films are fabricated and measured to develop the calibration curve. c) A linearly changing Fermi level is achieved depositing $20 \mathrm{~nm}$ thick films with the $\mathrm{R}=0 \%, 1.7 \%, 3.8 \%, 8.4 \%$, and $100 \%$. d) A ratchet style Fermi level across a $250 \mathrm{~nm}$ thick NC film is achieved by selecting three energy-equidistant mixing ratios, deduced from the calibration curve in panel b). 


\section{Supporting Information:}

SI 1) Electrolyte concentration optimization, SI 2) Note on Fermi level pinning and band bending in NC thin films immersed in electrolyte, SI 3) Discussion of substrate work functions, SI 4) Estimation of surface vacuum level shift based upon single electron charge transfer, SI 5) Reported bandstructures in NC thin films, SI 6) Photograph of measurement setup integrated in fabrication line, SI 7) Impact of electrolyte exposure on measured Fermi level, SI 8) Electrolyte impact on device performance, SI 9) Discussion of contact metallurgy

\section{Author Information:}

Corresponding author: *Email: vwood@ethz.ch

Author Contributions: SV performed the experiments. OY and MY synthesized the material. NY performed DFT simulations. SV, DB and VW envisioned the experiments. The manuscript was written through contributions of all authors.

Notes: The authors declare no financial interest.

\section{Acknowledgements}

The authors gratefully acknowledge funding from the Swiss National Science Foundation through Sinergia (project CRSII2_147615) and the NCCR Quantum Science and Technology. 
(1) Kim, J. Y.; Voznyy, O.; Zhitomirsky, D.; Sargent, E. H. 25th Anniversary Article: Colloidal Quantum Dot Materials and Devices: A Quarter-Century of Advances. $A d v$. Mater. 2013, 25, 4986-5010.

(2) Kovalenko, M. V.; Manna, L.; Cabot, A.; Hens, Z.; Talapin, D. V.; Kagan, C. R.; Klimov, V. I.; Rogach, A. L.; Reiss, P.; Milliron, D. J.; et al. Prospects of Nanoscience with Nanocrystals. ACS Nano 2015, 9, 1012-1057.

(3) Kroupa, D. M.; Vörös, M.; Brawand, N. P.; McNichols, B. W.; Miller, E. M.; Gu, J.; Nozik, A. J.; Sellinger, A.; Galli, G.; Beard, M. C. Tuning Colloidal Quantum Dot Band Edge Positions through Solution-Phase Surface Chemistry Modification. Nat. Commun. 2017, 8, 2-9.

(4) Brown, P. R.; Kim, D.; Lunt, R. R.; Zhao, N.; Bawendi, M. G.; Grossman, J. C.; Bulović, V. Energy Level Modification in Lead Sulfide Quantum Dot Thin Films through Ligand Exchange. ACS Nano 2014, 8, 5863-5872.

(5) Chuang, C. M.; Brown, P. R.; Bulović, V.; Bawendi, M. G. Improved Performance and Stability in Quantum Dot Solar Cells through Band Alignment Engineering. Nat. Mater. 2014, 13, 796-801.

(6) Ning, Z.; Voznyy, O.; Pan, J.; Hoogland, S.; Adinolfi, V.; Xu, J.; Li, M.; Kirmani, A. R.; Sun, J.-P.; Minor, J.; et al. Air-Stable n-Type Colloidal Quantum Dot Solids. Nat. Mater. 2014, 13, 822-828.

(7) Kahn, A. Fermi Level, Work Function and Vacuum Level. Mater. Horiz. 2016, 3, 7-10.

(8) Cahen, D.; Kahn, A. Electron Energetics at Surfaces and Interfaces: Concepts and Experiments. Adv. Mater. 2003, 15, 271-277.

(9) Ishii, H.; Sugiyama, K.; Ito, E.; Seki, K. Energy Level Alignment and Interfacial 
Electronic Structures at Organic/Metal and Organic/Organic Interfaces. Adv. Mater. 1999, 11, 605-625.

(10) Peljo, P.; Manzanares, J. A.; Girault, H. H. Contact Potentials, Fermi Level Equilibration, and Surface Charging. Langmuir 2016, 32, 5765-5775.

(11) Osella, S.; Cornil, D.; Cornil, J. Work Function Modification of the (111) Gold Surface Covered by Long Alkanethiol-Based Self-Assembled Monolayers. Phys. Chem. Chem. Phys. 2014, 16, 2866.

(12) Campbell, I.; Rubin, S.; Zawodzinski, T.; Kress, J.; Martin, R.; Smith, D.; Barashkov, N.; Ferraris, J. Controlling Schottky Energy Barriers in Organic Electronic Devices Using Self-Assembled Monolayers. Phys. Rev. B 1996, 54, 14321-14324.

(13) Alloway, D. M.; Hofmann, M.; Smith, D. L.; Gruhn, N. E.; Graham, A. L.; Colorado, R.; Wysocki, V. H.; Lee, T. R.; Lee, P. a; Armstrong, N. R. Interface Dipoles Arising from Self-Assembled Monolayers on Gold: UV-Photoemission Studies of Alkanethiols and Partially Fluorinated Alkanethiols. J. Phys. Chem. B 2003, 107, 11690-11699.

(14) Evans, S. D.; Urankar, E.; Ulman, A.; Ferris, N. Self-Assembled Monolayers of Alkanethiols Containing a Polar Aromatic Group: Effects of the Dipole Position on Molecular Packing, Orientation, and Surface Wetting Properties. J. Am. Chem. Soc. 1991, $113,4121-4131$.

(15) Evans, S. D.; Ulman, A. Surface Potential Studies of Alkyl-Thiol Monolayers Adsorbed on Gold. Chem. Phys. Lett. 1990, 170, 462-466.

(16) Zehner, R. W.; Parsons, B. F.; Hsung, R. P.; Sita, L. R. Tuning the Work Function of Gold with Self-Assembled Monolayers Derived from $\mathrm{X}-\left[\mathrm{C}_{6} \mathrm{H}_{4}-\mathrm{C}: \mathrm{C}-\right]_{\mathrm{n}} \mathrm{C}_{6} \mathrm{H}_{4}-\mathrm{SH}(\mathrm{n}=0,1,2 ; \mathrm{X}$ $=\mathrm{H}, \mathrm{F}, \mathrm{CH}_{3}, \mathrm{CF}_{3}$, and $\mathrm{OCH}_{3}$. Langmuir 1999, 15, 1121-1127. 
(17) De Boer, B.; Hadipour, A.; Mandoc, M. M.; Van Woudenbergh, T.; Blom, P. W. M. Tuning of Metal Work Functions with Self-Assembled Monolayers. Adv. Mater. 2005, 17, $621-625$.

(18) Crisp, R. W.; Kroupa, D. M.; Marshall, A. R.; Miller, E. M.; Zhang, J.; Beard, M. C.; Luther, J. M. Metal Halide Solid-State Surface Treatment for High Efficiency PbS and PbSe QD Solar Cells. Sci. Rep. 2015, 5, 9945.

(19) Soreni-Harari, M.; Yaacobi-Gross, N.; Steiner, D.; Aharoni, A.; Banin, U.; Millo, O.; Tessler, N. Tuning Energetic Levels in Nanocrystal Quantum Dots through Surface Manipulations. Nano Lett. 2008, 8, 678-684.

(20) Steiner, D.; Dorfs, D.; Banin, U.; Sala, F. D.; Manna, L.; Millo, O. Determination of Band Offsets in Heterostructured Colloidal Nanorods Using Scanning Tunneling Spectroscopy. Nano Lett. 2008, 8, 2954-2958.

(21) Dissanayake, D. M. N. M.; Lutz, T.; Curry, R. J.; Silva, S. R. P. Measurement and Validation of PbS Nanocrystal Energy Levels. Appl. Phys. Lett. 2008, 93, 91-94.

(22) Haram, S. K.; Quinn, B. M.; Bard, A. J. Electrochemistry of CdS Nanoparticles: A Correlation between Optical and Electrochemical Band Gaps. J. Am. Chem. Soc. 2001, $123,8860-8861$.

(23) Kucur, E.; Riegler, J.; Urban, G. a.; Nann, T. Determination of Quantum Confinement in CdSe Nanocrystals by Cyclic Voltammetry. J. Chem. Phys. 2003, 119, 2333-2337.

(24) Liu, J.; Yang, W.; Li, Y.; Fan, L.; Li, Y. Electrochemical Studies of the Effects of the Size, Ligand and Composition on the Band Structures of CdSe, CdTe and Their Alloy Nanocrystals. Phys. Chem. Chem. Phys. 2014, 16, 4778-4788.

(25) Volk, S.; Yazdani, N.; Sanusoglu, E.; Yarema, O.; Yarema, M.; Wood, V. Measuring the 
Electronic Structure of Nanocrystal Thin Films Using Energy-Resolved Electrochemical Impedance Spectroscopy. J. Phys. Chem. Lett. 2018, 1384-1392.

(26) Bisquert, J.; Fabregat-Santiago, F.; Mora-Seró, I.; Garcia-Belmonte, G.; Barea, E. M.; Palomares, E. A Review of Recent Results on Electrochemical Determination of the Density of Electronic States of Nanostructured Metal-Oxide Semiconductors and Organic Hole Conductors. Inorganica Chim. Acta 2008, 361, 684-698.

(27) Wang, H.; Wang, Y.; He, B.; Li, W.; Sulaman, M.; Xu, J.; Yang, S.; Tang, Y.; Zou, B. Charge Carrier Conduction Mechanism in PbS Quantum Dot Solar Cells: Electrochemical Impedance Spectroscopy Study. ACS Appl. Mater. Interfaces 2016, 8, 18526-18533.

(28) Bloom, B. P.; Zhao, L. Bin; Wang, Y.; Waldeck, D. H.; Liu, R.; Zhang, P.; Beratan, D. N. Ligand-Induced Changes in the Characteristic Size-Dependent Electronic Energies of CdSe Nanocrystals. J. Phys. Chem. C 2013, 117, 22401-22411.

(29) Sobrova, P.; Ryvolova, M.; Hubalek, J.; Adam, V.; Kizek, R. Voltammetry as a Tool for Characterization of CdTe Quantum Dots. Int. J. Mol. Sci. 2013, 14, 13497-13510.

(30) Kagan, C. R.; Lifshitz, E.; Sargent, E. H.; Talapin, D. V. Building Devices from Colloidal Quantum Dots. Science 2016, 353, 5523.

(31) Haynes, W. M. CRC Handbook of Chemistry and Physics; Taylor \& Francis: New York, 2008.

(32) Schlaf, R.; Murata, H.; Kafafi, Z. H. Work Function Measurements on Indium Tin Oxide Films. J. Electron Spectros. Relat. Phenomena 2001, 120, 149-154.

(33) Shrotriya, V.; Li, G.; Yao, Y.; Chu, C.-W.; Yang, Y. Transition Metal Oxides as the Buffer Layer for Polymer Photovoltaic Cells. Appl. Phys. Lett. 2006, 88, 073508.

(34) Hung, L. S.; Tang, C. W.; Mason, M. G. Enhanced Electron Injection in Organic 
Electroluminescence Devices Using an Al/LiF Electrode. Appl. Phys. Lett. 1997, 70, 152154.

(35) Brabec, C. J.; Shaheen, S. E.; Winder, C.; Sariciftci, N. S.; Denk, P. Effect of LiF/Metal Electrodes on the Performance of Plastic Solar Cells. Appl. Phys. Lett. 2002, 80, 12881290.

(36) Tang, J.; Wang, X.; Brzozowski, L.; Barkhouse, D. A. R.; Debnath, R.; Levina, L.; Sargent, E. H. Schottky Quantum Dot Solar Cells Stable in Air under Solar Illumination. Adv. Mater. 2010, 22, 1398-1402.

(37) Bullock, J.; Hettick, M.; Geissbühler, J.; Ong, A. J.; Allen, T.; Sutter-Fella, C. M.; Chen, T.; Ota, H.; Wolf, S. De; Schaler, E. W.; et al. Efficient Silicon Solar Cells with DopantFree Asymmetric Heterocontacts. Nat. Energy 2016, 1, 1-7.

(38) Sun, Z.; Shi, S.; Bao, Q.; Liu, X.; Fahlman, M. Role of Thick-Lithium Fluoride Layer in Energy Level Alignment at Organic/Metal Interface: Unifying Effect on High Metallic Work Functions. Adv. Mater. Interfaces 2015, 2, 1400527.

(39) Heimel, G.; Romaner, L.; Zojer, E.; Bredas, J. L. Toward Control of the Metal-Organic Interfacial Electronic Structure in Molecular Electronics: A First-Principles Study on SelfAssembled Monolayers of $\pi$-Conjugated Molecules on Noble Metals. Nano Lett. 2007, 7, 932-940.

(40) Saraswathi, N.; Soundararajan, S. Charge Distribution, Dipole Moment and Infrared Spectra of Some Thiol and Thio Acids. J. Mol. Struct. 1968, 4, 419-433.

(41) Karle, J.; Brockway, L. O. An Electron Diffraction Investigation of the Monomers and Dimers of Formic, Acetic and Trifluoroacetic Acids and the Dimer of Deuterium Acetate. J. Am. Chem. Soc. 1944, 66, 574-584. 
(42) Doan, V.; Köppe, R.; Kasai, P. H. Dimerization of Carboxylic Acids and Salts: An IR Study in Perfluoropolyether Media. J. Am. Chem. Soc. 1997, 119, 9810-9815.

(43) Serr, A.; Netz, R. R. Polarizabilities of Hydrated and Free Ions Derived From DFT Calculations. Int. J. Quantum Chem. 2006, 105, 2960-2974.

(44) Diaconescu, B.; Padilha, L. A.; Nagpal, P.; Swartzentruber, B. S.; Klimov, V. I. Measurement of Electronic States of PbS Nanocrystal Quantum Dots Using Scanning Tunneling Spectroscopy: The Role of Parity Selection Rules in Optical Absorption. Phys. Rev. Lett. 2013, 110, 1-5.

(45) Ciszek, J. W.; Tour, J. M. Mechanistic Implications of the Assembly of Organic Thiocyanates on Precious Metals. Chem. Mater. 2005, 17, 5684-5690.

(46) Itabashi, E.; Ikeda, S. Catalytic Polarographic Wave of Nickel(II) in Aqueous Thiocyanate Solutions Containing Tetraethylammonium Ions. J. Electroanal. Chem. 1970, 27, 243256.

(47) Krogulec, T.; Baranski, A.; Galus, Z. On the Formation of Sulphides in the Electroreduction of Thiocyanate Complexes of Nickel(II) at Mercury Electrodes. 1974, $57,63-75$.

(48) Houmam, A.; Hamed, E. M.; Still, I. W. J. A Unique Autocatalytic Process and Evidence for a Concerted-Stepwise Mechanism Transition in the Dissociative Electron-Transfer Reduction of Aryl Thiocyanates. J. Am. Chem. Soc. 2003, 125, 7258-7265.

Oh, S. J.; Berry, N. E.; Choi, J. H.; Gaulding, E. A.; Lin, H.; Paik, T.; Diroll, B. T.; Muramoto, S.; Murray, C. B.; Kagan, C. R. Designing High-Performance PbS and PbSe Nanocrystal Electronic Devices through Stepwise, Post-Synthesis, Colloidal Atomic Layer Deposition. Nano Lett. 2014, 14, 1559-1566. 
Oh, S. J.; Berry, N. E.; Choi, J. H.; Gaulding, E. A.; Paik, T.; Hong, S. H.; Murray, C. B.; Kagan, C. R. Stoichiometric Control of Lead Chalcogenide Nanocrystal Solids to Enhance Their Electronic and Optoelectronic Device Performance. ACS Nano 2013, 7, 2413-2421.

(51) Voznyy, O.; Zhitomirsky, D.; Stadler, P.; Ning, Z.; Hoogland, S.; Sargent, E. H. A Charge-Orbital Balance Picture of Doping in Colloidal Quantum Dot Solids. ACS Nano 2012, 6, 8448-8455.

(52) Luther, J.; Law, M.; Song, Q.; Perkins, C. Structural, Optical, and Electrical Properties of Self-Assembled Films of PbSe Nanocrystals Treated with 1, 2-Ethanedithiol. ACS Nano 2008, 2, 271-280.

(53) Grosso, D. How to Exploit the Full Potential of the Dip-Coating Process to Better Control Film Formation. J. Mater. Chem. 2011, 21, 17033.

(54) Zhao, N.; Osedach, T. P.; Chang, L.-Y.; Geyer, S. M.; Wanger, D.; Binda, M. T.; Arango, A. C.; Bawendi, M. G.; Bulovic, V. Colloidal PbS Quantum Dot Solar Cells with High Fill Factor. ACS Nano 2010, 4, 3743-3752.

(55) Hines, M. a.; Scholes, G. D. Colloidal PbS Nanocrystals with Size-Tunable Near-Infrared Emission: Observation of Post-Synthesis Self-Narrowing of the Particle Size Distribution. Adv. Mater. 2003, 15, 1844-1849.

(56) Trasatti, S. The Absolute Electrode Potential: An Explanatory Note. Pure Appl. Chem. 1986, $58,955-966$.

(57) Trasatti, S. The "Absolute" Electrode Potential-the End of the Story. Electrochim. Acta 1990, 35, 269-271.

(58) BioLogic Science Instruments: Reference electrodes \& porous glass frits http://www.biologic.net/en/accessories/electrodes/reference-electrodes-porous-glass-frits/ (accessed Dec 
15, 2017).

(59) Reiss, H.; Heller, A. The Absolute Potential of the Standard Hydrogen Electrode: A New Estimate. J. Phys. Chem. 1985, 89, 4207-4213.

(60) Weidman, M. C.; Yager, K. G.; Tisdale, W. A. Interparticle Spacing and Structural Ordering in Superlattice Pbs Nanocrystal Solids Undergoing Ligand Exchange. Chem. Mater. 2015, 27, 474-482.

(61) Yazdani, N.; Nguyen-Thanh, T.; Yarema, M.; Lin, W. M. M.; Gao, R.; Yarema, O.; Bosak, A.; Wood, V. Measuring the Vibrational Density of States of Nanocrystal-Based Thin Films with Inelastic X-Ray Scattering. J. Phys. Chem. Lett. 2018, 9, 1561-1567.

(62) Yazdani, N.; Bozyigit, D.; Vuttivorakulchai, K.; Luisier, M.; Infante, I.; Wood, V. Tuning Electron-Phonon Interactions in Nanocrystals through Surface Termination. Nano Lett. 2018, 18, 2233-2242.

(63) Zherebetskyy, D.; Scheele, M.; Zhang, Y.; Bronstein, N.; Thompson, C.; Britt, D.; Salmeron, M.; Alivisatos, P.; Wang, L.-W. Hydroxylation of the Surface of PbS Nanocrystals Passivated with Oleic Acid. Science 2014, 346, 1396-1402.

(64) Vandevondele, J.; Krack, M.; Mohamed, F.; Parrinello, M.; Chassaing, T.; Hutter, J. Quickstep: Fast and Accurate Density Functional Calculations Using a Mixed Gaussian and Plane Waves Approach. Comput. Phys. Commun. 2005, 167, 103-128.

(65) Lippert, B. G.; Parrinello, J. H. and M. A Hybrid Gaussian and Plane Wave Density Functional Scheme. Mol. Phys. 1997, 92, 477-488.

(66) VandeVondele, J.; Hutter, J. Gaussian Basis Sets for Accurate Calculations on Molecular Systems in Gas and Condensed Phases. J. Chem. Phys. 2007, 127, 114105.

(67) Hartwigsen, C.; Goedecker, S.; Hutter, J. Relativistic Separable Dual-Space Gaussian 
Pseudopotentials from H to Rn. Phys. Rev. B 1998, 58, 3641-3662.

(68) Genovese, L.; Deutsch, T.; Neelov, A.; Goedecker, S.; Beylkin, G. Efficient Solution of Poisson's Equation with Free Boundary Conditions. J. Chem. Phys. 2006, 125, 074105.

(69) Genovese, L.; Deutsch, T.; Goedecker, S. Efficient and Accurate Three-Dimensional Poisson Solver for Surface Problems. J. Chem. Phys. 2007, 127, 054704. 